\title{
Analysis of Segregated Boundary-Domain Integral Equations for Variable-Coefficient Problems with Cracks
}

\author{
O. Chkadua ${ }^{1}$, S.E. Mikhailov ${ }^{2 *}$ and D. Natroshvili ${ }^{3}$ \\ ${ }^{1}$ A.Razmadze Mathematical Institute, Georgian Acad. of Sci., and Sokhumi State University, \\ Tbilisi, Georgia \\ ${ }^{2}$ Department of Mathematics, Brunel University, UK \\ ${ }^{3}$ Dept. of Mathematics, Georgian Technical University and I. Vekua Institute of Applied \\ Mathematics of Tbilisi State University, Georgia
}

\begin{abstract}
Segregated direct boundary-domain integral equation (BDIE) systems associated with mixed, Dirichlet and Neumann boundary value problems (BVPs) for a scalar "Laplace" PDE with variable coefficient are formulated and analysed for domains with interior cuts (cracks). The main results established in the paper are the BDIE equivalence to the original BVPs and invertibility of the BDIE operators in the corresponding Sobolev spaces.
\end{abstract}

Keywords: Partial Differential Equation, Variable coefficients, Boundary-Domain Integral Equations.

\section{Introduction}

Partial Differential Equations (PDEs) with variable coefficients arise naturally in mathematical modelling of non-homogeneous media (e.g. functionally graded materials or materials with damage induced inhomogeneity) in solid mechanics, electro-magnetics, thermo-conductivity, fluid flows trough porous media, and other areas of physics and engineering.

The Boundary Integral Equation Method (Boundary Element Method) is a well established tool for solution Boundary Value Problems (BVPs) with constant coefficients. The main ingredient for reducing a BVP for a PDE to a BIE is a fundamental solution to the original PDE, see e.g. $[6,10,8]$. However, it is generally not available in an analytical and/or cheaply calculated form for PDEs with variable coefficients. Following Levi and Hilbert, one can use in this case a parametrix (Levi function) as a substitute for the fundamental solution. Parametrix is usually much wider available than a fundamental solution and correctly describes the main part of the fundamental solution although does not have to satisfy the original PDE. This reduces the problem not to a boundary integral equation but to a Boundary-Domain Integral Equation (BDIE) system, see e.g. $[15,16]$.

In this paper, extending approach of $[2,3]$, we develop analysis of direct segregated BDIEs for the Dirichlet, Neumann and mixed variable-coefficient BVPs in domains with interior cuts (cracks), whose faces are subject to the Neumann conditions. Our main goal is to prove

(i) equivalence of the BDIE to the original crack type BVPs and

(ii) invertibility of the corresponding boundary-domain integral operators in appropriate Sobolev (Bessel potential) spaces.

\footnotetext{
${ }^{*}$ Corresponding author: e-mail: sergey.mikhailov@brunel.ac.uk, Phone: +44 189267361 , Fax: +441895269732
} 


\section{Formulation of the boundary value problems}

Let $\Omega=\Omega^{+}$be a bounded open three-dimensional region of $\mathbb{R}^{3}$ and $\Omega^{-}:=\mathbb{R}^{3} \backslash \bar{\Omega}$. For simplicity, we assume that the boundary $\partial \Omega$ is a simply connected, closed, infinitely smooth surface. Moreover, $\partial \Omega=\bar{S}_{D} \cup \bar{S}_{N}$ where $S_{D}$ and $S_{N}$ are nonintersecting $\left(S_{D} \cap S_{N}=\varnothing\right)$, simply connected sub-manifolds of $\partial \Omega$ with infinitely smooth boundary curve $\ell:=\partial S_{D}=$ $\partial S_{N} \in C^{\infty}$. If either $S_{D}=\varnothing$ or $S_{N}=\varnothing$, then $\ell=\varnothing$. Further, we assume that the region $\Omega$ contains an interior crack. We define the crack as a two-dimensional, two-sided open manifold $\Sigma$ with the crack edge $\partial \Sigma$. We assume that $\Sigma$ is a sub-manifold of a simply connected closed infinitely smooth surface $\partial \Omega_{0} \subset \Omega$ which is the boundary of a domain $\bar{\Omega}_{0} \subset \Omega$. Denote by $\Omega_{\Sigma}:=\Omega \backslash \bar{\Sigma}$ the domain with crack. Throughout the paper $n=\left(n_{1}, n_{2}, n_{3}\right)$ stands for the unit normal vector to $\partial \Omega$ exterior to $\Omega$ and for the unit normal vector to $\partial \Omega_{0}$ exterior to $\Omega_{0}$. This agreement defines the positive direction of the normal vector on the crack surface $\Sigma$.

Further, let $a \in C^{\infty}(\bar{\Omega}), a(x)>0$ for $x \in \bar{\Omega}$. Let also $\partial_{j}=\partial_{x_{j}}:=\partial / \partial x_{j}(j=1,2,3)$, $\partial_{x}=\left(\partial_{x_{1}}, \partial_{x_{2}}, \partial_{x_{3}}\right)$. We consider boundary-domain integral equations associated with the following scalar elliptic differential equation

$$
L u(x):=L\left(x, \partial_{x}\right) u(x):=\sum_{i=1}^{3} \partial_{x_{i}}\left(a(x) \partial_{x_{i}} u(x)\right)=f(x), \quad x \in \Omega_{\Sigma},
$$

where $u$ is an unknown function and $f$ is a given function in $\Omega_{\Sigma}$.

In what follows $H^{s}(\Omega)=H_{2}^{s}(\Omega), H^{s}(\partial \Omega)=H_{2}^{s}(\partial \Omega), H^{s}\left(\partial \Omega_{0}\right)=H_{2}^{s}\left(\partial \Omega_{0}\right), s \in \mathbb{R}$, denote the Sobolev-Slobodetski (the Bessel potential) spaces, while $H^{1}\left(\Omega_{\Sigma}\right)=W_{2}^{1}\left(\Omega_{\Sigma}\right)$ is the Sobolev space for the domain with crack. For $S \subset \partial \Omega$, we will use the space $\widetilde{H}^{s}(S)=$ $\left\{g: g \in H^{s}(\partial \Omega)\right.$, supp $\left.g \subset \bar{S}\right\}$, and the space $H^{s}(S)=\left\{r_{S} g: g \in H^{s}(\partial \Omega)\right\}$ of restriction on $S$ of functions from $H^{s}(\partial \Omega)$, where $r_{S}$ denotes the restriction operator on $S$. Similar spaces are defined also on $\Sigma \subset \partial \Omega_{0}$.

From the trace theorem (see, e.g., [9]) it follows that $\gamma^{+} u \in H^{\frac{1}{2}}(\partial \Omega), \gamma^{ \pm} u \in H^{\frac{1}{2}}(\Sigma)$ for $u \in H^{1}\left(\Omega_{\Sigma}\right)$, where $\gamma^{ \pm}$is the trace operator.

For $u \in H^{2}\left(\Omega_{\Sigma}\right)$, we denote by $T^{ \pm}$the corresponding co-normal derivative operator on $\partial \Omega$ and $\Sigma$ in the trace sense,

$$
T^{ \pm} u(x):=a(x) \partial_{n}^{ \pm} u(x):=\sum_{i=1}^{3} a(x) n_{i}(x) \gamma^{ \pm}\left[\partial_{i} u(x)\right],
$$

where $\partial_{n}$ denotes the corresponding normal derivative operator. If $T^{+} u=T^{-} u$, we will write Tu.

For the linear operator $L$, we introduce the following subspace of $H^{1}\left(\Omega_{\Sigma}\right)$, c.f. $[7,5,13]$,

$$
H^{1,0}\left(\Omega_{\Sigma} ; L\right):=\left\{g: g \in H^{1}\left(\Omega_{\Sigma}\right), L g \in L_{2}\left(\Omega_{\Sigma}\right)\right\}
$$

endowed with the norm

$$
\|g\|_{H^{1,0}\left(\Omega_{\Sigma} ; L\right)}^{2}:=\|g\|_{H^{1}\left(\Omega_{\Sigma}\right)}^{2}+\|L g\|_{L_{2}\left(\Omega_{\Sigma}\right)}^{2} .
$$

For a couple of functions $\left(g^{+}, g^{-}\right)$defined on the surface $\Sigma$, we denote their difference (jump) as $[g]_{\Sigma}:=g^{+}-g^{-}$, their average as $g_{\Sigma}^{0}:=\left(g^{+}+g^{-}\right) / 2$, and introduce the space

$$
\mathbb{H}^{s}(\Sigma):=\left\{\left(g^{+}, g^{-}\right): g_{\Sigma}^{0} \in H^{s}(\Sigma),[g]_{\Sigma} \in \tilde{H}^{s}(\Sigma)\right\} .
$$

For $u \in H^{1}\left(\Omega_{\Sigma}\right)$ the co-normal derivatives on $\partial \Omega$ and $\Sigma$ do not generally exist in the trace sense. However if $u \in H^{1,0}\left(\Omega_{\Sigma} ; L\right)$, one can correctly define the generalized (canonical) 
co-normal derivatives $\left(T^{+} u, T^{-} u\right) \in \mathbb{H}^{-\frac{1}{2}}(\Sigma),\left(T^{+} u \in H^{-\frac{1}{2}}(\partial \Omega)\right.$, similar to [7, Theorem 1.5.3.10], [5, Lemma 3.2], [14, Definition 3], as

$$
\begin{gathered}
T^{ \pm} u:=T_{\Sigma}^{0} u \pm \frac{1}{2}[T]_{\Sigma} u \quad \text { on } \Sigma \\
\left\langle T^{+} u, w_{\partial \Omega}\right\rangle_{\partial \Omega}+\left\langle[T]_{\Sigma} u, w_{\Sigma}^{0}\right\rangle_{\Sigma}+\left\langle T_{\Sigma}^{0} u,\left[w_{\Sigma}\right]_{\Sigma}\right\rangle_{\Sigma}:=\int_{\Omega_{\Sigma}}\left[\gamma_{-1} w L u+E\left(u, \gamma_{-1} w\right)\right] d x \\
\forall w=\left\{w_{\partial \Omega},\left(w_{\Sigma}^{+}, w_{\Sigma}^{-}\right)\right\} \in H^{\frac{1}{2}}(\partial \Omega) \times \mathbb{H}^{\frac{1}{2}}(\Sigma) .
\end{gathered}
$$

If $\mu u \in H^{1,0}\left(\Omega^{-} ; L\right)$ for any $\mu \in C_{\text {comp }}^{\infty}\left(\overline{\Omega^{-}}\right)$, then

$$
\left\langle T^{-} u, w_{\partial \Omega}\right\rangle_{\partial \Omega}:=-\int_{\Omega^{-}}\left[\gamma_{-1}^{-} w L u+E\left(u, \gamma_{-1}^{-} w\right)\right] d x \quad \forall w=w_{\partial \Omega} \in H^{\frac{1}{2}}(\partial \Omega) .
$$

Here $\gamma_{-1}: H^{\frac{1}{2}}(\partial \Omega) \times \mathbb{H}^{\frac{1}{2}}(\Sigma) \rightarrow H^{1}\left(\Omega_{\Sigma}\right)$ and $\gamma_{-1}^{-}: H^{\frac{1}{2}}(\partial \Omega) \rightarrow H_{\text {com }}^{1}\left(\Omega^{-}\right)$are continuous right inverse operators to the trace operators,

$$
E(u, v):=\sum_{i=1}^{3} a(x) \partial_{i} u(x) \partial_{i} v(x)
$$

$\langle\cdot, \cdot\rangle_{\partial \Omega}$ denotes the duality brackets between the spaces $H^{-s}(\partial \Omega)$ and $H^{s}(\partial \Omega),\langle\cdot, \cdot\rangle_{\Sigma}$ the duality brackets between the spaces $H^{-s}(\Sigma)$ and $\tilde{H}^{s}(\Sigma), s \in \mathbb{R}$, which extend the usual $L_{2}(\partial \Omega)$ and $L_{2}(\Sigma)$ inner products. We also used the notation

$$
\left\langle T^{+} u, w_{\Sigma}^{+}\right\rangle_{\Sigma}-\left\langle T^{-} u, w_{\Sigma}^{-}\right\rangle_{\Sigma}:=\left\langle[T]_{\Sigma} u, w_{\Sigma}^{0}\right\rangle_{\Sigma}+\left\langle T_{\Sigma}^{0} u,\left[w_{\Sigma}\right]_{\Sigma}\right\rangle_{\Sigma},
$$

which is well defined for $T^{ \pm} u \in \mathbb{H}^{-s}(\Sigma), w_{\Sigma}^{ \pm} \in \mathbb{H}^{s}(\Sigma), s \in \mathbb{R}$.

Similar to [7, Theorem 1.5.3.11], [5, Lemma 3.4], [14, Definition 3], one can prove that the co-normal derivatives do not depend on the choice of the operator $\gamma_{-1}$, and the first Green identity

$$
\int_{\Omega_{\Sigma}}[v L u+E(u, v)] d x=\left\langle T^{+} u, \gamma^{+} v\right\rangle_{S}+\left\langle T^{+} u, \gamma^{+} v\right\rangle_{\Sigma}-\left\langle T^{-} u, \gamma^{-} v\right\rangle_{\Sigma},
$$

holds for any functions $u \in H^{1,0}\left(\Omega_{\Sigma} ; L\right), v \in H^{1}\left(\Omega_{\Sigma}\right)$, while the second Green identity

$$
\begin{aligned}
& \int_{\Omega_{\Sigma}}[v L u-L v u] d x=\left\langle T^{+} u, \gamma^{+} v\right\rangle_{\partial \Omega}-\left\langle T^{+} v, \gamma^{+} u\right\rangle_{\partial \Omega} \\
&+\left\langle T^{+} u, \gamma^{+} v\right\rangle_{\Sigma}-\left\langle T^{-} u, \gamma^{-} v\right\rangle_{\Sigma}+\left\langle T^{+} v, \gamma^{+} u\right\rangle_{\Sigma}-\left\langle T^{-} v, \gamma^{-} u\right\rangle_{\Sigma}
\end{aligned}
$$

holds for any functions $u, v \in H^{1,0}\left(\Omega_{\Sigma} ; L\right)$.

We will consider the BDIE approach for the following three crack type boundary value problems.

Mixed BVP with crack, or Problem (MC): Find a function $u \in H^{1}\left(\Omega_{\Sigma}\right)$ satisfying the conditions

$$
\begin{aligned}
& L u=f \text { in } \Omega_{\Sigma}, \\
& r_{S_{D}} \gamma^{+} u=\varphi_{0} \text { on } S_{D},
\end{aligned}
$$




$$
\begin{aligned}
& r_{S_{N}} T^{+} u=\psi_{0} \text { on } S_{N}, \\
& T^{+} u=\psi_{\Sigma}^{+}, \quad T^{-} u=\psi_{\Sigma}^{-} \text {on } \Sigma,
\end{aligned}
$$

where

$$
\varphi_{0} \in H^{\frac{1}{2}}\left(S_{D}\right), \quad \psi_{0} \in H^{-\frac{1}{2}}\left(S_{N}\right), \quad\left(\psi_{\Sigma}^{+}, \psi_{\Sigma}^{-}\right) \in \mathbb{H}^{-\frac{1}{2}}(\Sigma), \quad f \in H^{0}\left(\Omega_{\Sigma}\right) .
$$

Note that we can replace the crack conditions (2.11) by the equivalent ones,

$$
[T]_{\Sigma} u=\left[\psi_{\Sigma}\right]_{\Sigma}, \quad T_{\Sigma}^{0} u=\psi_{\Sigma}^{0} \quad \text { on } \quad \Sigma .
$$

Equation (2.8) is understood in the distributional sense, condition (2.9) in the trace sense, while equality (2.10) and (2.11) in the functional sense (2.3)-(2.4).

Clearly, if $S_{N}=\varnothing$ in (2.8)-(2.11), we arrive at the Dirichlet problem with crack, or Problem (DC): Find $u \in H^{1}\left(\Omega_{\Sigma}\right)$ such that

$$
\begin{aligned}
& L u=f \quad \text { in } \quad \Omega_{\Sigma}, \\
& \gamma^{+} u=\varphi_{0} \text { on } \partial \Omega, \\
& T^{+} u=\psi_{\Sigma}^{+}, \quad T^{-} u=\psi_{\Sigma}^{-} \text {on } \Sigma .
\end{aligned}
$$

where

$$
\varphi_{0} \in H^{\frac{1}{2}}(\partial \Omega), \quad\left(\psi_{\Sigma}^{+}, \psi_{\Sigma}^{-}\right) \in \mathbb{H}^{-\frac{1}{2}}(\Sigma), \quad f \in H^{0}\left(\Omega_{\Sigma}\right) .
$$

If $S_{D}=\varnothing$ in (2.8)-(2.11), we have the Neumann problem with crack, or Problem (NC): Find $u \in H^{1}\left(\Omega_{\Sigma}\right)$ such that

$$
\begin{aligned}
& L u=f \quad \text { in } \quad \Omega_{\Sigma}, \\
& T^{+} u=\psi_{0} \text { on } \partial \Omega, \\
& T^{+} u=\psi_{\Sigma}^{+}, \quad T^{-} u=\psi_{\Sigma}^{-} \text {on } \Sigma .
\end{aligned}
$$

where

$$
\psi_{0} \in H^{-\frac{1}{2}}(\partial \Omega), \quad\left(\psi_{\Sigma}^{+}, \psi_{\Sigma}^{-}\right) \in \mathbb{H}^{-\frac{1}{2}}(\Sigma), \quad f \in H^{0}\left(\Omega_{\Sigma}\right) .
$$

We have (similar e.g. to [9, Chapter 2, Section 9]) the following well-known uniqueness and existence result.

THEOREM 2.1 (i) The homogeneous Dirichlet and mixed BVPs with crack have only the trivial solution, while the homogeneous Neumann crack problem admits a constant as a general solution.

(ii) The nonhomogeneous problem (DC) under condition (2.17), and the nonhomogeneous problem (MC) under condition (2.12) are uniquely solvable. if

(iii) Let the inclusions (2.21) be satisfied. Then the problem (NC) is solvable if and only

$$
\int_{\Omega_{\Sigma}} f(x) d x=\int_{\partial \Omega} \psi_{0}(x) d S+\int_{\Sigma}\left[\psi_{\Sigma}^{+}(x)-\psi_{\Sigma}^{-}(x)\right] d S,
$$

and the solution $u$ is defined modulo constant summand.

Proof. The uniqueness results immediately follow from the first Green identity (2.6) with $v=u$ as a solution of the corresponding homogeneous boundary value problem. The existence results directly follow from the Lax-Milgram theorem applied to the weak variational formulation of the above problems.

In the subsequent sections our main goal is to reduce the above BVPs to the equivalent boundary-domain integral (pseudodifferential) equations and to prove invertibility of the corresponding nonstandard integral operators in appropriate function spaces. 


\section{Surface and volume potentials and the third Green identity}

The function

$$
P(x, y)=-\frac{1}{4 \pi a(y)|x-y|}, \quad x, y \in \mathbb{R}^{3}, \quad x \neq y,
$$

is a parametrix (Levi function) of the operator $L\left(x, \partial_{x}\right)$ with the property

$$
L\left(x, \partial_{x}\right) P(x, y)=\delta(x-y)+R(x, y),
$$

where $\delta(\cdot)$ is the Dirac distribution and the remainder

$$
R(x, y)=\sum_{i=1}^{3} \frac{x_{i}-y_{i}}{4 \pi a(y)|x-y|^{3}} \frac{\partial a(x)}{\partial x_{i}}, \quad x, y \in \mathbb{R}^{3}, x \neq y,
$$

possesses a weak singularity of type $\mathcal{O}\left(|x-y|^{-2}\right)$ for small $|x-y|$, see $[11,2]$.

Further we introduce parametrix-based surface potential operators

$$
\begin{aligned}
& V_{\partial \Omega} g(y):=-\int_{\partial \Omega} P(x, y) g(x) d S_{x}, \quad y \in \mathbb{R}^{3} \backslash \partial \Omega, \\
& W_{\partial \Omega} g(y):=-\int_{\partial \Omega}\left[T_{x} P(x, y)\right] g(x) d S_{x}, \quad y \in \mathbb{R}^{3} \backslash \partial \Omega, \\
& V_{\Sigma} g(y):=-\int_{\Sigma} P(x, y) g(x) d S_{x}, \quad y \in \mathbb{R}^{3} \backslash \Sigma, \\
& W_{\Sigma} g(y):=-\int_{\Sigma}\left[T_{x} P(x, y)\right] g(x) d S_{x}, \quad y \in \mathbb{R}^{3} \backslash \Sigma,
\end{aligned}
$$

and volume potential operators

$$
\mathcal{P} g(y):=\int_{\Omega_{\Sigma}} P(x, y) g(x) d x, \quad \mathcal{R} g(y):=\int_{\Omega_{\Sigma}} R(x, y) g(x) d x, \quad y \in \mathbb{R}^{3} .
$$

The corresponding direct values of the surface potentials are denoted as

$$
\begin{aligned}
& \mathcal{V}_{\partial \Omega} g(y):=-\int_{\partial \Omega} P(x, y) g(x) d S_{x}, \quad y \in \partial \Omega, \\
& \mathcal{W}_{\partial \Omega} g(y):=-\int_{\partial \Omega}\left[T_{x} P(x, y)\right] g(x) d S_{x}, \quad y \in \partial \Omega, \\
& \mathcal{V}_{\Sigma} g(y):=-\int_{\Sigma} P(x, y) g(x) d S_{x}, \quad y \in \Sigma, \\
& \mathcal{W}_{\Sigma} g(y):=-\int_{\Sigma}\left[T_{x} P(x, y)\right] g(x) d S_{x}, \quad y \in \Sigma,
\end{aligned}
$$

and the co-normal derivatives of the surface potentials as

$$
\begin{aligned}
& \mathcal{W}_{\partial \Omega}^{\prime} g(y):=-\int_{\partial \Omega}\left[T_{y} P(x, y)\right] g(x) d S_{x}, \quad \mathcal{L}_{\partial \Omega}^{ \pm} g(y):=T^{ \pm} W_{\partial \Omega} g(y), \quad y \in \partial \Omega, \\
& \mathcal{W}_{\Sigma}^{\prime} g(y):=-\int_{\Sigma}\left[T_{y} P(x, y)\right] g(x) d S_{x}, \quad \mathcal{L}_{\Sigma}^{ \pm} g(y):=T^{ \pm} W_{\Sigma} g(y), \quad y \in \Sigma, \\
& {[\mathcal{L}]_{\Sigma} g(y):=\mathcal{L}_{\Sigma}^{+} g(y)-\mathcal{L}_{\Sigma}^{-} g(y), \quad \mathcal{L}_{\Sigma}^{0} g(y):=\frac{1}{2}\left\{\mathcal{L}_{\Sigma}^{+} g(y)+\mathcal{L}_{\Sigma}^{-} g(y)\right\}, \quad y \in \Sigma .}
\end{aligned}
$$


Mapping and jump properties of operators (3.4)-(3.9) in Sobolev spaces are given in [2] (see also the Appendix below). Particularly, by Theorems A.3 and B.1 of the Appendix, for any $f \in H^{0}\left(\Omega_{\Sigma}\right), u \in H^{1}\left(\Omega_{\Sigma}\right), \varphi^{*} \in \widetilde{H}^{\frac{1}{2}}(\Sigma)$ we have,

$$
\begin{aligned}
{[\mathcal{P} f]_{\Sigma}=0, \quad[T]_{\Sigma} \mathcal{P} f=0, \quad[\mathcal{R} u]_{\Sigma}=0, } \\
\quad[T]_{\Sigma} \mathcal{R} u=-\left(\partial_{n} a\right)[u]_{\Sigma}, \quad[\mathcal{L}]_{\Sigma} \varphi^{*}=\left(\partial_{n} a\right) \varphi^{*} \quad \text { on } \quad \Sigma,
\end{aligned}
$$

where $[u]_{\Sigma}:=\gamma^{+} u-\gamma^{-} u$ on $\Sigma$.

Taking, as in $[11,2], v(x):=P(x, y)$ and $u \in H^{1,0}\left(\Omega_{\Sigma} ; L\right)$ in $(2.7)$, we obtain by the standard limiting procedures (see e.g. [15]) the third Green identity,

$$
u+\mathcal{R} u-V_{\partial \Omega}\left(T^{+} u\right)+W_{\partial \Omega}\left(\gamma^{+} u\right)-V_{\Sigma}\left([T]_{\Sigma} u\right)+W_{\Sigma}\left([u]_{\Sigma}\right)=\mathcal{P} L u \quad \text { in } \quad \Omega_{\Sigma} .
$$

Taking trace of (3.11) and its co-normal derivative on $\partial \Omega$ and the average of its co-normal derivatives, $T_{\Sigma}^{0}=\frac{1}{2}\left(T^{+}+T^{-}\right)$, on $\Sigma$, we obtain,

$$
\begin{aligned}
& \frac{1}{2} \gamma^{+} u+\gamma^{+} \mathcal{R} u-\mathcal{V}_{\partial \Omega} T^{+} u+\mathcal{W}_{\partial \Omega} \gamma^{+} u-\gamma^{+} V_{\Sigma}[T]_{\Sigma} u+\gamma^{+} W_{\Sigma}[u]_{\Sigma} \\
& =\gamma^{+} \mathcal{P} L u \quad \text { on } \quad \partial \Omega, \\
& \frac{1}{2} T^{+} u+T^{+} \mathcal{R} u-\mathcal{W}_{\partial \Omega}^{\prime} T^{+} u+\mathcal{L}_{\partial \Omega}^{+} \gamma^{+} u-T^{+} V_{\Sigma}[T]_{\Sigma} u+T^{+} W_{\Sigma}[u]_{\Sigma} \\
& =T^{+} \mathcal{P} L u \quad \text { on } \quad \partial \Omega \text {, } \\
& T_{\Sigma}^{0} u+T_{\Sigma}^{0} \mathcal{R} u-T_{\Sigma}^{0} V_{\partial \Omega} T^{+} u+T_{\Sigma}^{0} W_{\partial \Omega} \gamma^{+} u-\mathcal{W}_{\Sigma}^{\prime}[T]_{\Sigma} u+\mathcal{L}_{\Sigma}^{0}[u]_{\Sigma} \\
& =T_{\Sigma}^{0} \mathcal{P} L u \quad \text { on } \quad \Sigma .
\end{aligned}
$$

The co-normal derivatives in the last two equations are well defined due to the inclusion of each term of $(3.11)$ in $H^{1,0}\left(\Omega_{\Sigma} ; L\right)$ by Theorems A.1 and B.1.

\section{Segregated BDIEs for the problem (MC)}

To get a segregated boundary domain integral formulation for the problem (MC), we replace the unknown traces, co-normal derivatives and jumps of $u$ on $S_{N}, S_{D}$ and $\Sigma$ with new unknown functions that will be treated as independent of $u$. First of all, we denote $\varphi^{*}:=$ $[u]_{\Sigma} \in \widetilde{H}^{\frac{1}{2}}(\Sigma)$. Let now $\Phi_{0} \in H^{\frac{1}{2}}(\partial \Omega)$ be a fixed extension of the given right hand side of the Dirichlet condition (2.9), $\varphi_{0} \in H^{\frac{1}{2}}\left(S_{D}\right)$, onto the whole of $\partial \Omega$. Then $\gamma^{+} u=\Phi_{0}+\varphi$ on $\partial \Omega$, where the unknown function $\varphi$ belongs to $\widetilde{H}^{\frac{1}{2}}\left(S_{N}\right)$ due to (2.9). Analogously, let $\Psi_{0} \in H^{-\frac{1}{2}}(\partial \Omega)$ be a fixed extension of the given right hand side of the Neumann condition (2.10), $\psi_{0} \in H^{-\frac{1}{2}}\left(S_{D}\right)$, onto the whole of $\partial \Omega$. Then $T^{+} u=\Psi_{0}+\psi$, where the unknown function $\psi$ belongs to $\widetilde{H}^{-\frac{1}{2}}\left(S_{D}\right)$ due to (2.10). (If $\varphi_{0}=0$ or $\psi_{0}=0$ then we can take the canonical extensions $\Phi_{0}=0$ or $\Psi_{0}=0$, respectively, on $\partial \Omega$.) By this way we have introduced the following unknown functions,

$$
\psi=T^{+} u-\Psi_{0} \in \widetilde{H}^{-\frac{1}{2}}\left(S_{D}\right), \varphi=\gamma^{+} u-\Phi_{0} \in \widetilde{H}^{\frac{1}{2}}\left(S_{N}\right), \quad \varphi^{*}=[u]_{\Sigma} \in \widetilde{H}^{\frac{1}{2}}(\Sigma) .
$$

Let

$$
\mathbb{X}:=H^{1}\left(\Omega_{\Sigma}\right) \times \widetilde{H}^{-\frac{1}{2}}\left(S_{D}\right) \times \widetilde{H}^{\frac{1}{2}}\left(S_{N}\right) \times \widetilde{H}^{\frac{1}{2}}(\Sigma),
$$

We show below, similar to [2], that if $u \in H^{1,0}\left(\Omega_{\Sigma} ; L\right)$ is a solution of the problem (MC) then the four-vector $\mathcal{U}=\left(u, \psi, \varphi, \varphi^{*}\right)^{\top} \in \mathbb{X}$ satisfies four different systems of BDIEs. 
BDIE system (MC11). Taking (3.11) in the domain, (3.12) on $S_{D},(3.13)$ on $S_{N},(3.14)$ on $\Sigma$, substituting boundary/crack conditions (2.9)-(2.11) and employing relations (4.1), we derive the segregated boundary-domain integral equation system (MC11) of four equations for $\left(u, \psi, \varphi, \varphi^{*}\right) \in \mathbb{X}$,

$$
\begin{aligned}
u+\mathcal{R} u-V_{\partial \Omega} \psi+W_{\partial \Omega} \varphi+W_{\Sigma} \varphi^{*} & =F_{0} & \text { in } \Omega_{\Sigma}, \\
r_{S_{D}}\left\{\gamma^{+} \mathcal{R} u-\mathcal{V}_{\partial \Omega} \psi+\mathcal{W}_{\partial \Omega} \varphi+\gamma^{+} W_{\Sigma} \varphi^{*}\right\} & =r_{S_{D}} \gamma^{+} F_{0}-\varphi_{0} & \text { on } S_{D}, \\
r_{S_{N}}\left\{T^{+} \mathcal{R} u-\mathcal{W}_{\partial \Omega}^{\prime} \psi+\mathcal{L}_{\partial \Omega}^{+} \varphi+T^{+} W_{\Sigma} \varphi^{*}\right\} & =r_{S_{N}} T^{+} F_{0}-\psi_{0} & \text { on } S_{N}, \\
T_{\Sigma}^{0} \mathcal{R} u-T_{\Sigma}^{0} V_{\partial \Omega} \psi+T_{\Sigma}^{0} W_{\partial \Omega} \varphi+\mathcal{L}_{\Sigma}^{0} \varphi^{*} & =T_{\Sigma}^{0} F_{0}-\psi_{\Sigma}^{0} & \text { on } \Sigma,
\end{aligned}
$$

where

$$
F_{0}:=\mathcal{P} f+V_{\Sigma}\left[\psi_{\Sigma}\right]_{\Sigma}+V_{\partial \Omega} \Psi_{0}-W_{\partial \Omega} \Phi_{0} \text { in } \Omega_{\Sigma} .
$$

The notation (MC11) indicates that the BDIE system includes integral operators (4.4) and (4.5) of the first kind on the Dirichlet and Neumann parts of the boundary, respectively. Denote the $4 \times 4$ matrix operator generated by the left hand side of the BDIE system (MC11) as

$$
\mathcal{A}^{11}:=\left[\begin{array}{cccc}
I+\mathcal{R} & -V_{\partial \Omega} & W_{\partial \Omega} & W_{\Sigma} \\
r_{S_{D}} \gamma^{+} \mathcal{R} & -r_{S_{D}} \mathcal{V}_{\partial \Omega} & r_{S_{D}} \mathcal{W}_{\partial \Omega} & r_{S_{D}} \gamma^{+} W_{\Sigma} \\
r_{S_{N}} T^{+} \mathcal{R} & -r_{S_{N}} \mathcal{W}_{\partial \Omega}^{\prime} & r_{S_{N}} \mathcal{L}_{\partial \Omega}^{+} & r_{S_{N}} T^{+} W_{\Sigma} \\
T_{\Sigma}^{0} \mathcal{R} & -T_{\Sigma}^{0} V_{\partial \Omega} & T_{\Sigma}^{0} W_{\partial \Omega} & \mathcal{L}_{\Sigma}^{0}
\end{array}\right]
$$

where $I$ is the identity operator in corresponding spaces. The system can be rewritten as

$$
\mathcal{A}^{11} \mathcal{U}=\mathcal{F}^{11}
$$

where

$$
\mathcal{F}^{11} \in \mathbb{F}^{11}:=H^{1}\left(\Omega_{\Sigma}\right) \times H^{\frac{1}{2}}\left(S_{D}\right) \times H^{-\frac{1}{2}}\left(S_{N}\right) \times H^{-\frac{1}{2}}(\Sigma)
$$

BDIE system (MC12). Taking again (3.11) in the domain, but (3.12) on the whole of $\partial \Omega,(3.14)$ on $\Sigma$, substituting boundary/crack conditions (2.9)-(2.11) and employing relations (4.1), we derive the segregated boundary-domain integral equation system (MC12) of three equations for $\left(u, \psi, \varphi, \varphi^{*}\right) \in \mathbb{X}$,

$$
\begin{array}{rlr}
u+\mathcal{R} u-V_{\partial \Omega} \psi+W_{\partial \Omega} \varphi+W_{\Sigma} \varphi^{*} & =F_{0} & \text { in } \Omega_{\Sigma}, \\
\frac{1}{2} \varphi+\gamma^{+} \mathcal{R} u-\mathcal{V}_{\partial \Omega} \psi+\mathcal{W}_{\partial \Omega} \varphi+\gamma^{+} W_{\Sigma} \varphi^{*} & =\gamma^{+} F_{0}-\Phi_{0} & \text { on } \partial \Omega, \\
T_{\Sigma}^{0} \mathcal{R} u-T_{\Sigma}^{0} V_{\partial \Omega} \psi+T_{\Sigma}^{0} W_{\partial \Omega} \varphi+\mathcal{L}_{\Sigma}^{0} \varphi^{*} & =T_{\Sigma}^{0} F_{0}-\psi_{\Sigma}^{0} & \text { on } \Sigma .
\end{array}
$$

The notation (MC12) indicates that the BDIE system includes integral operator of the third kind (4.10) on $\partial \Omega$, which is of the first kind on $S_{D}$ (since $\varphi=0$ on $S_{D}$ due to the inclusion $\left.\varphi \in \widetilde{H}^{\frac{1}{2}}\left(S_{N}\right)\right)$ and of the second kind on $S_{N}$. Denoting the $3 \times 4$ matrix operator generated by the left hand side of the BDIE system (MC12) as

$$
\mathcal{A}^{12}:=\left[\begin{array}{cccc}
I+\mathcal{R} & -V_{\partial \Omega} & W_{\partial \Omega} & W_{\Sigma} \\
\gamma^{+} \mathcal{R} & -\mathcal{V}_{\partial \Omega} & \frac{1}{2} I+\mathcal{W}_{\partial \Omega} & \gamma^{+} W_{\Sigma} \\
T_{\Sigma}^{0} \mathcal{R} & -T_{\Sigma}^{0} V_{\partial \Omega} & T_{\Sigma}^{0} W_{\partial \Omega} & \mathcal{L}_{\Sigma}^{0}
\end{array}\right],
$$


the system can be rewritten as

$$
\mathcal{A}^{12} \mathcal{U}=\mathcal{F}^{12}
$$

where

$$
\mathcal{F}^{12} \in \mathbb{F}^{12}:=H^{1}\left(\Omega_{\Sigma}\right) \times H^{\frac{1}{2}}(\partial \Omega) \times H^{-\frac{1}{2}}(\Sigma) .
$$

BDIE system (MC21). To obtain the third BDIE system, we take, as before, (3.11) in the domain, but (3.13) on $\partial \Omega,(3.14)$ on $\Sigma$, substituting boundary/crack conditions (2.9)(2.11) and employing relations (4.1), we derive the boundary-domain integral equation system (MC21) of three equations for $\left(u, \psi, \varphi, \varphi^{*}\right) \in \mathbb{X}$,

$$
\begin{array}{rlr}
u+\mathcal{R} u-V_{\partial \Omega} \psi+W_{\partial \Omega} \varphi+W_{\Sigma} \varphi^{*} & =F_{0} & \text { in } \Omega_{\Sigma}, \\
\frac{1}{2} \psi+T^{+} \mathcal{R} u-\mathcal{W}_{\partial \Omega}^{\prime} \psi+\mathcal{L}_{\partial \Omega}^{+} \varphi+T^{+} W_{\Sigma} \varphi^{*} & =T^{+} F_{0}-\Psi_{0} & \text { on } \partial \Omega, \\
T_{\Sigma}^{0} \mathcal{R} u-T_{\Sigma}^{0} V_{\partial \Omega} \psi+T_{\Sigma}^{0} W_{\partial \Omega} \varphi+\mathcal{L}_{\Sigma}^{0} \varphi^{*} & =T_{\Sigma}^{0} F_{0}-\psi_{\Sigma}^{0} & \text { on } \Sigma .
\end{array}
$$

The integral operator (4.14) is of the third kind, i.e., it is of the second kind on $S_{D}$ and of the first kind on $S_{N}$ (since $\psi=0$ on $S_{N}$ due to the inclusion $\psi \in \widetilde{H}^{\frac{1}{2}}\left(S_{D}\right)$ ). Denoting the $3 \times 4$ matrix operator generated by the left hand side of the BDIE system (MC21) as

$$
\mathcal{A}^{21}:=\left[\begin{array}{cccc}
I+\mathcal{R} & -V_{\partial \Omega} & W_{\partial \Omega} & W_{\Sigma} \\
T^{+} \mathcal{R} & \frac{1}{2} I-\mathcal{W}_{\partial \Omega}^{\prime} & \mathcal{L}_{\partial \Omega}^{+} & T^{+} W_{\Sigma} \\
T_{\Sigma}^{0} \mathcal{R} & -T_{\Sigma}^{0} V_{\partial \Omega} & T_{\Sigma}^{0} W_{\partial \Omega} & \mathcal{L}_{\Sigma}^{0}
\end{array}\right],
$$

the system can be rewritten as

$$
\mathcal{A}^{21} \mathcal{U}=\mathcal{F}^{21}
$$

where

$$
\mathcal{F}^{21} \in \mathbb{F}^{21}:=H^{1}\left(\Omega_{\Sigma}\right) \times H^{-\frac{1}{2}}(\partial \Omega) \times H^{-\frac{1}{2}}(\Sigma)
$$

BDIE system (MC22). At last, we take (3.11) in the domain, (3.13) on $S_{D},(3.12)$ on $S_{N}$, (3.14) on $\Sigma$, substitute boundary/crack conditions (2.9)-(2.11) and employ relations (4.1), to derive the boundary-domain integral equation system (MC22) of four equations for $\left(u, \psi, \varphi, \varphi^{*}\right) \in \mathbb{X}$

$$
\begin{aligned}
u+\mathcal{R} u-V_{\partial \Omega} \psi+W_{\partial \Omega} \varphi+W_{\Sigma} \varphi^{*} & =F_{0} \quad \text { in } \Omega_{\Sigma}, \\
\frac{1}{2} \psi+r_{S_{D}}\left\{T^{+} \mathcal{R} u-\mathcal{W}_{\partial \Omega}^{\prime} \psi+\mathcal{L}_{\partial \Omega}^{+} \varphi+T^{+} W_{\Sigma} \varphi^{*}\right\} & =r_{S_{D}} T^{+} F_{0}-r_{S_{D}} \Psi_{0} \quad \text { on } S_{D}, \\
\frac{1}{2} \varphi+r_{S_{N}}\left\{\gamma^{+} \mathcal{R} u-\mathcal{V}_{\partial \Omega} \psi+\mathcal{W}_{\partial \Omega} \varphi+\gamma^{+} W_{\Sigma} \varphi^{*}\right\} & =r_{S_{N}} \gamma^{+} F_{0}-r_{S_{N}} \Phi_{0} \quad \text { on } S_{N}, \\
T_{\Sigma}^{0} \mathcal{R} u-T_{\Sigma}^{0} V_{\partial \Omega} \psi+T_{\Sigma}^{0} W_{\partial \Omega} \varphi+\mathcal{L}_{\Sigma}^{0} \varphi^{*} & =T_{\Sigma}^{0} F_{0}-\psi_{\Sigma}^{0} \quad \text { on } \Sigma .
\end{aligned}
$$

The notation (MC22) indicates that the BDIE system includes integral operators (4.18) and (4.19) of the second kind on the Dirichlet and Neumann parts of the boundary, respectively. Denoting the $4 \times 4$ matrix operator generated by the left hand side of the BDIE system 
$(\mathrm{MC} 22)$ as

$$
\mathcal{A}^{22}:=\left[\begin{array}{cccc}
I+\mathcal{R} & -V_{\partial \Omega} & W_{\partial \Omega} & W_{\Sigma} \\
r_{S_{D}} T^{+} \mathcal{R} & r_{S_{D}}\left(\frac{1}{2} I-\mathcal{W}_{\partial \Omega}^{\prime}\right) & r_{S_{D}} \mathcal{L}_{\partial \Omega}^{+} & r_{S_{D}} T^{+} W_{\Sigma} \\
r_{S_{N}} \gamma^{+} \mathcal{R} & -r_{S_{N}} \mathcal{V}_{\partial \Omega} & r_{S_{N}}\left(\frac{1}{2} I+\mathcal{W}_{\partial \Omega}\right) & r_{S_{N}} \gamma^{+} W_{\Sigma} \\
T_{\Sigma}^{0} \mathcal{R} & -T_{\Sigma}^{0} V_{\partial \Omega} & T_{\Sigma}^{0} W_{\partial \Omega} & \mathcal{L}_{\Sigma}^{0}
\end{array}\right],
$$

the system can be rewritten as

$$
\mathcal{A}^{22} \mathcal{U}=\mathcal{F}^{22}
$$

where

$$
\mathcal{F}^{22} \in \mathbb{F}^{22}:=H^{1}\left(\Omega_{\Sigma}\right) \times H^{-\frac{1}{2}}\left(S_{D}\right) \times H^{\frac{1}{2}}\left(S_{N}\right) \times H^{-\frac{1}{2}}(\Sigma) .
$$

Now we prove the basic equivalence theorem for the problem (MC) and BDIE systems (MC11), (MC12), (MC21), (MC22).

THEOREM 4.1 Let conditions (2.12) hold and let $\Phi_{0} \in H^{\frac{1}{2}}(\partial \Omega)$ and $\Psi_{0} \in H^{-\frac{1}{2}}(\partial \Omega)$ be some extensions of $\varphi_{0}$ and $\psi_{0}$, respectively.

(i) If a function $u \in H^{1}\left(\Omega_{\Sigma}\right)$ solves the problem $(M C)$, then the four-vector $\left(u, \psi, \varphi, \varphi^{*}\right)$, where $\psi, \varphi$, and $\varphi^{*}$ are defined by (4.1), solves the BDIE systems (MC11), (MC12), (MC21) and (MC22).

(ii) If a four-vector $\left(u, \psi, \varphi, \varphi^{*}\right) \in H^{1}\left(\Omega_{\Sigma}\right) \times \widetilde{H}^{-\frac{1}{2}}\left(S_{D}\right) \times \widetilde{H}^{\frac{1}{2}}\left(S_{N}\right) \times \widetilde{H}^{\frac{1}{2}}(\Sigma)$ solves one of the BDIE systems (MC11), (MC12), (MC21) or (MC22), then this solution is unique and solves all the systems, while u solves the problem (MC) and relations (4.1) hold.

Proof. For a function $u \in H^{1}\left(\Omega_{\Sigma}\right)$ being a solution to (2.8) under conditions (2.12) we have $u \in H^{1,0}\left(\Omega_{\Sigma} ; L\right)$ since $f \in H^{0}\left(\Omega^{+}\right)$. Under hypothesis of item (i) this implies (3.11) and thus the claims of item (i) for all the BDIE systems.

Now, let a four-vector $\left(u, \psi, \varphi, \varphi^{*}\right) \in H^{1}\left(\Omega_{\Sigma}\right) \times \widetilde{H}^{-\frac{1}{2}}\left(S_{D}\right) \times \widetilde{H}^{\frac{1}{2}}\left(S_{N}\right) \times \widetilde{H}^{\frac{1}{2}}(\Sigma)$ solve the BDIE system (MC11). Theorems A.1 and B.1 and the first equation of the system, (4.3), imply that all its terms belong to $H^{1,0}\left(\Omega_{\Sigma} ; L\right)$ and thus their co-normal derivatives are well defined. Similar to the proof of Theorem 5.2 in [2] for the corresponding BDIE system without crack, we show that $u$ solves the problem (MC).

From (4.4) and the trace of (4.3) on $\partial \Omega$ we conclude that $r_{S_{D}} u^{+}=\varphi_{0}$ on $S_{D}$, while from (4.5) and the co-normal derivative of (4.3) on $\partial \Omega$ we have $r_{S_{N}} T^{+} u=\psi_{0}$ on $S_{N}$. Taking the jump of traces of (4.3) on $\Sigma$ we get

$$
[u]_{\Sigma}=\varphi^{*} \text { on } \Sigma .
$$

Further, take the co-normal derivatives $T^{+}, T^{-}$of the equation (4.3) on $\Sigma$, construct their difference, and compare their sum with (4.6) to obtain

$$
r_{\Sigma}\left\{T^{+} u-T^{-} u-[u]_{\Sigma} \partial_{n} a-\left(\left[\psi_{\Sigma}\right]_{\Sigma}\right)+\varphi^{*} \partial_{n} a\right\}=0, \quad r_{\Sigma}\left\{T^{+} u+T^{-} u\right\}=2 \psi_{\Sigma}^{0},
$$

i.e.,

$$
[T]_{\Sigma} u=\left[\psi_{\Sigma}\right]_{\Sigma}, \quad T_{\Sigma}^{0} u=\psi_{\Sigma}^{0} \text { on } \quad \Sigma .
$$

These relations coincide with (2.13) thus implying (2.11).

As already mentioned, $u \in H^{1,0}\left(\Omega_{\Sigma} ; L\right)$, and we can write Green's third identity (3.11) for $u$. Comparing it with equation (4.3) and taking into account (4.22), (4.23) gives

$$
-V_{\partial \Omega}\left(T^{+} u-\psi-\Psi_{0}\right)+W_{\partial \Omega}\left(u^{+}-\varphi-\Phi_{0}\right)=\mathcal{P}(L u-f) \text { in } \Omega_{\Sigma} .
$$


Since all the potentials in (4.24) are continuous on $\Omega$ (including $\Sigma$ ), equation (4.24) can be extended on the whole $\Omega$. Then taking into account that $u^{+}-\varphi-\Phi_{0}=0$ on $S_{D}$ and $T^{+} u-\psi-\Psi_{0}=0$ on $S_{N}$, we obtain by [2, Lemmas 4.1,4.2] that $L u-f=0$ in $\Omega$, while $u^{+}-\varphi-\Phi_{0}=0$ and $T^{+} u-\psi-\Psi_{0}=0$ on $\partial \Omega$. By item (i) of the theorem this implies that the four-vector $\left(u, \psi, \varphi, \varphi^{*}\right)$ solves also all the BDIE systems.

We now have to prove uniqueness of solution of the BDIE system (MC11). Let $\left(u, \psi, \varphi, \varphi^{*}\right) \in$ $H^{1}\left(\Omega_{\Sigma}\right) \times \widetilde{H}^{\frac{1}{2}}\left(S_{N}\right) \times \widetilde{H}^{-\frac{1}{2}}\left(S_{D}\right) \times \widetilde{H}^{\frac{1}{2}}(\Sigma)$ solve homogeneous BDIE system (4.3)-(4.6), which zero right hand side can be considered as generated by the zero right hand side of problem $(\mathrm{MC}),\left(\varphi_{0}, \psi_{0}, \psi_{\Sigma}^{ \pm}, f\right)=0$. Then already proved statements of item (ii) imply that $u$ is a solution of the homogeneous problem (MC), which is zero by Theorem 2.1, and thus $\left(\psi, \varphi, \varphi^{*}\right)=0$ by item (i).

The proof of item (ii) for the BDIE systems (MC12), (MC21) and (MC22) follows the same pattern and uses the similarity with the proofs of the equivalence Theorems 5.6, 5.9, 5.12 in [2] for the corresponding BDIE systems without crack.

Further we study invertibility in appropriate function spaces of the matrix operators $\mathcal{A}^{11}$, $\mathcal{A}^{12}, \mathcal{A}^{21}$ and $\mathcal{A}^{22}$.

In view of the mapping properties of the potential type operators (3.4)-(3.8), see Appendix, the operators

$$
\mathcal{A}^{\alpha \beta}: \mathbb{X} \rightarrow \mathbb{F}^{\alpha \beta}
$$

are continuous for any $\alpha, \beta=1,2$. By Theorem 4.1(ii) all the operators (4.25) are injective. Moreover, we are now in the position to prove the following assertion.

THEOREM 4.2 The operators $\mathcal{A}^{\alpha \beta}: \mathbb{X} \rightarrow \mathbb{F}^{\alpha \beta}$ are continuous and continuously invertible for any $\alpha, \beta=1,2$.

Proof. The proof will follow the pattern of the proofs for the corresponding operators without crack in [2].

Note that we have the identity (see [2, Theorem 3.6])

$$
\mathcal{L}_{S}^{ \pm} g=\hat{\mathcal{L}}_{S} g+\left(\partial_{n} a\right)\left( \pm \frac{1}{2} g-\mathcal{W}_{S} g\right)=\mathcal{L}_{S}^{0} g \pm \frac{1}{2} g \partial_{n} a,
$$

with either $S=\partial \Omega$ or $S=\Sigma$. Here

$$
\hat{\mathcal{L}}_{S} g:=\mathcal{L}_{S, \Delta}(a g)=\left[T W_{S, \Delta}(a g)\right]^{+}=\left[T W_{S, \Delta}(a g)\right]^{-} \quad \text { on } S,
$$

where $W_{S, \Delta}(a g)$ is the usual harmonic double layer potential over $S$ with density $a g$,

$$
W_{S, \Delta}(a g)(y)=\frac{1}{4 \pi} \int_{S} \frac{\partial}{\partial n(x)} \frac{1}{|x-y|} a(x) g(x) d S_{x} .
$$

Equality (4.26) then represents the well-known Liapunov-Tauber theorem for a harmonic double layer potential.

First, let us consider the operator

$$
\mathcal{A}_{0}^{11}:=\left[\begin{array}{cccc}
I & -V_{\partial \Omega} & W_{\partial \Omega} & W_{\Sigma} \\
0 & -r_{S_{D}} \mathcal{V}_{\partial \Omega} & 0 & 0 \\
0 & 0 & r_{S_{N}} \hat{\mathcal{L}}_{\partial \Omega} & 0 \\
0 & 0 & 0 & \hat{\mathcal{L}}_{\Sigma}
\end{array}\right] .
$$

As follows from Appendix, the operator $\mathcal{A}_{0}^{11}: \mathbb{X} \rightarrow \mathbb{F}^{11}$ is continuous and is a compact perturbation to the operator $\mathcal{A}^{11}: \mathbb{X} \rightarrow \mathbb{F}^{11}$. 
Since the diagonal operators

$$
\begin{aligned}
r_{S_{D}} \mathcal{V}_{\partial \Omega} & : \quad \widetilde{H}^{-\frac{1}{2}}\left(S_{D}\right) \rightarrow H^{\frac{1}{2}}\left(S_{D}\right), \\
r_{S_{N}} \hat{\mathcal{L}}_{\partial \Omega} & : \quad \widetilde{H}^{\frac{1}{2}}\left(S_{N}\right) \rightarrow H^{-\frac{1}{2}}\left(S_{N}\right), \\
\hat{\mathcal{L}}_{\Sigma} & : \quad \widetilde{H}^{\frac{1}{2}}(\Sigma) \rightarrow H^{-\frac{1}{2}}(\Sigma),
\end{aligned}
$$

are invertible (see Theorems A.4, A.5), we conclude that the triangular operator $\mathcal{A}_{0}^{11}: \mathbb{X} \rightarrow$ $\mathbb{F}^{11}$ is invertible, implying that (4.25) is a Fredholm operator with index zero. Therefore from injectivity of $\mathcal{A}^{11}: \mathbb{X} \rightarrow \mathbb{F}^{11}$ follows its invertibility.

To analyse operator $\mathcal{A}^{21}$ let us consider the auxiliary operator

$$
\mathcal{A}_{0}^{21}:=\left[\begin{array}{cccc}
I & -V_{\partial \Omega} & W_{\partial \Omega} & W_{\Sigma} \\
0 & \frac{1}{2} I & \hat{\mathcal{L}}_{\partial \Omega} & 0 \\
0 & 0 & 0 & \hat{\mathcal{L}}_{\Sigma}
\end{array}\right],
$$

which is continuous and is a compact perturbation to the operator $\mathcal{A}^{21}: \mathbb{X} \rightarrow \mathbb{F}^{21}$, see Appendix. Any solution $\mathcal{U} \in \mathbb{X}$ of the equation $\mathcal{A}_{0}^{21} \mathcal{U}=\mathcal{F}^{21}$, where $\mathcal{F}^{21}=\left(\mathcal{F}_{1}^{21}, \mathcal{F}_{2}^{21}, \mathcal{F}_{3}^{21}\right)^{\top} \in$ $H^{1}\left(\Omega_{\Sigma}\right) \times H^{-\frac{1}{2}}(\partial \Omega) \times H^{-\frac{1}{2}}(\Sigma)$ will solve also the equation $\mathcal{A}_{*}^{21} \mathcal{U}=\mathcal{F}_{*}^{21}$, where

$$
\mathcal{A}_{*}^{21}:=\left[\begin{array}{cccc}
I & -V_{\partial \Omega} & W_{\partial \Omega} & W_{\Sigma} \\
0 & \frac{1}{2} I & \hat{\mathcal{L}}_{\partial \Omega} & 0 \\
0 & 0 & r_{S_{N}} \hat{\mathcal{L}}_{\partial \Omega} & 0 \\
0 & 0 & 0 & \hat{\mathcal{L}}_{\Sigma}
\end{array}\right]
$$

$\mathcal{F}_{*}^{21}=\left(\mathcal{F}_{1}^{21}, \mathcal{F}_{2}^{21}, r_{S_{N}} \mathcal{F}_{2}^{21}, \mathcal{F}_{3}^{21}\right)^{\top} \in \mathbb{F}_{*}^{21}:=H^{1}\left(\Omega_{\Sigma}\right) \times H^{-\frac{1}{2}}(\partial \Omega) \times H^{-\frac{1}{2}}\left(S_{N}\right) \times H^{-\frac{1}{2}}(\Sigma)$, and vice-versa. Since the diagonal operators (4.29), (4.30) are invertible, we conclude that the triangular operator $\mathcal{A}_{*}^{21}: \mathbb{X}_{*}^{21} \rightarrow \mathbb{F}_{*}^{21}$ is invertible, where $\mathbb{X}_{*}^{21}=H^{1}\left(\Omega_{\Sigma}\right) \times H^{-\frac{1}{2}}(\partial \Omega) \times$ $\widetilde{H}^{\frac{1}{2}}\left(S_{N}\right) \times \widetilde{H}^{\frac{1}{2}}(\Sigma)$. Moreover, if $\mathcal{U}$ is a solution of the system $\mathcal{A}_{*}^{21} \mathcal{U}=\mathcal{F}_{*}^{21}$, then subtracting the third equation of the system from restriction to $S_{N}$ of the second equation implies that $\psi=\mathcal{U}_{2}=0$ on $S_{N}$. That is, in fact $\mathcal{U}=\left(\mathcal{A}_{*}^{21}\right)^{-1} \mathcal{F}_{*}^{21} \in \mathbb{X}$, implying also invertibility of the operator $\mathcal{A}_{0}^{21}: \mathbb{X} \rightarrow \mathbb{F}^{21}$ and thus the Fredholm property with zero index for the operator $\mathcal{A}^{21}: \mathbb{X} \rightarrow \mathbb{F}^{21}$. Therefore from injectivity of $\mathcal{A}^{21}$ follows its invertibility.

Invertibility of the operator $\mathcal{A}^{12}: \mathbb{X} \rightarrow \mathbb{F}^{12}$ is proved similarly.

To analyse operator $\mathcal{A}^{22}$ let us consider the auxiliary operator

$$
\mathcal{A}_{0}^{22}:=\left[\begin{array}{cccc}
I & -V_{\partial \Omega} & W_{\partial \Omega} & W_{\Sigma} \\
0 & r_{S_{D}}\left(\frac{1}{2} I-\mathcal{W}_{\Delta, \partial \Omega}^{\prime}\right) & r_{S_{D}} \hat{\mathcal{L}}_{\partial \Omega} & 0 \\
0 & -r_{S_{N}} \mathcal{V}_{\partial \Omega} & r_{S_{N}}\left(\frac{1}{2} I+\mathcal{W}_{\partial \Omega}\right) & 0 \\
0 & 0 & 0 & \hat{\mathcal{L}}_{\Sigma}
\end{array}\right],
$$

which is continuous and is a compact perturbation to the operator $\mathcal{A}^{22}: \mathbb{X} \rightarrow \mathbb{F}^{22}$, see Appendix. The operator $\mathcal{A}_{0}^{22}$ can be considered as block-triangle operator with the middle diagonal block

$$
\hat{\mathcal{A}}_{0}^{22}:=\left[\begin{array}{cc}
r_{S_{D}}\left(\frac{1}{2} I-\mathcal{W}_{\Delta, \partial \Omega}^{\prime}\right) & r_{S_{D}} \hat{\mathcal{L}}_{\partial \Omega} \\
-r_{S_{N}} \mathcal{V}_{\partial \Omega} & r_{S_{N}}\left(\frac{1}{2} I+\mathcal{W}_{\partial \Omega}\right)
\end{array}\right],
$$


that can be presented as

$$
\begin{gathered}
\hat{\mathcal{A}}_{0}^{22} \hat{\mathcal{U}}=\operatorname{diag}\left(1, \frac{1}{a}\right) \hat{\mathcal{A}}_{\Delta}^{22}[\operatorname{diag}(1, a) \hat{\mathcal{U}}], \\
\hat{\mathcal{A}}_{\Delta}^{22}:=\left[\begin{array}{cc}
r_{S_{D}}\left(\frac{1}{2} I-\mathcal{W}_{\Delta, \partial \Omega}^{\prime}\right) & r_{S_{D}} \hat{\mathcal{L}}_{\Delta, \partial \Omega} \\
-r_{S_{N}} \mathcal{V}_{\Delta, \partial \Omega} & r_{S_{N}}\left(\frac{1}{2} I+\mathcal{W}_{\Delta, \partial \Omega}\right)
\end{array}\right] .
\end{gathered}
$$

The operator $\hat{\mathcal{A}}_{\Delta}^{22}: \widetilde{H}^{-\frac{1}{2}}\left(S_{D}\right) \times \widetilde{H}^{\frac{1}{2}}\left(S_{N}\right) \rightarrow H^{-\frac{1}{2}}\left(S_{D}\right) \times H^{\frac{1}{2}}\left(S_{N}\right)$ is invertible by [2, Theorem 5.18], which implies invertibility of the operator $\hat{\mathcal{A}}_{0}^{22}: \widetilde{H}^{-\frac{1}{2}}\left(S_{D}\right) \times \widetilde{H}^{\frac{1}{2}}\left(S_{N}\right) \rightarrow H^{-\frac{1}{2}}\left(S_{D}\right) \times$ $H^{\frac{1}{2}}\left(S_{N}\right)$ since $0<C_{1}<a<C_{2}<\infty$. Taking into account the invertibility of operator (4.30), we obtain invertibility of the operator $\mathcal{A}_{0}^{22}: \mathbb{X} \rightarrow \mathbb{F}^{22}$ and thus the Fredholm property with zero index for the operator $\mathcal{A}^{22}: \mathbb{X} \rightarrow \mathbb{F}^{22}$, whose injectivity then implies its invertibility.

\section{Segregated BDIEs for the problem (DC)}

Segregated BDIE systems for problem (DC) is formulated by the same way as for the problem (MC) but with apparent simplifications. Let $u \in H^{1,0}\left(\Omega_{\Sigma} ; L\right)$ be a solution of the problem (DC) and let us introduce the notations

$$
\psi=T^{+} u \in H^{-\frac{1}{2}}(\partial \Omega), \quad \varphi^{*}=[u]_{\Sigma} \in \widetilde{H}^{\frac{1}{2}}(\Sigma),
$$

for unknown boundary/crack values. Let $\mathbb{X}^{D}:=H^{1}\left(\Omega_{\Sigma}\right) \times H^{-\frac{1}{2}}(\partial \Omega) \times \widetilde{H}^{\frac{1}{2}}(\Sigma)$.

BDIE system (DC1). Taking (3.11) in the domain, (3.12) on $\partial \Omega,(3.14)$ on $\Sigma$, substituting boundary/crack conditions (2.15)-(2.16) and employing (5.1), we derive the following boundary-domain integral equation system $(\mathrm{DC} 1)$ for $\left(u, \psi, \varphi^{*}\right) \in \mathbb{X}^{D}$,

$$
\begin{array}{rlrl}
u+\mathcal{R} u-V_{\partial \Omega} \psi+W_{\Sigma} \varphi^{*} & =F_{0}^{D} & \text { in } \Omega_{\Sigma}, \\
\gamma^{+} \mathcal{R} u-\mathcal{V}_{\partial \Omega} \psi+\gamma^{+} W_{\Sigma} \varphi^{*} & =\gamma^{+} F_{0}^{D}-\varphi_{0} & & \text { on } \partial \Omega, \\
T_{\Sigma}^{0} \mathcal{R} u-T_{\Sigma}^{0} V_{\partial \Omega} \psi+\mathcal{L}_{\Sigma}^{0} \varphi^{*} & =T_{\Sigma}^{0} F_{0}^{D}-\psi_{\Sigma}^{0} & & \text { on } \Sigma,
\end{array}
$$

where

$$
F_{0}^{D}:=\mathcal{P} f+V_{\Sigma}\left(\left[\psi_{\Sigma}\right]_{\Sigma}\right)-W_{\partial \Omega} \varphi_{0} \text { in } \Omega_{\Sigma} .
$$

Let us denote the left hand side operator of the BDIE system (DC1) as

$$
\mathcal{A}^{D 1}:=\left[\begin{array}{ccc}
I+\mathcal{R} & -V_{\partial \Omega} & W_{\Sigma} \\
\gamma_{\partial \Omega}^{+} \mathcal{R} & -\mathcal{V}_{\partial \Omega} & \gamma_{\partial \Omega}^{+} W_{\Sigma} \\
T_{\Sigma}^{0} \mathcal{R} & -T_{\Sigma}^{0} V_{\partial \Omega} & \mathcal{L}_{\Sigma}^{0}
\end{array}\right],
$$

where $\gamma_{\partial \Omega}^{+}:=r_{\partial \Omega} \gamma^{+}$.

BDIE system (DC2). Taking (3.11) in the domain, but now (3.13) on $\partial \Omega$ and again (3.14) on $\Sigma$, substituting boundary/crack conditions (2.15)-(2.16) and employing (5.1), we derive the following boundary-domain integral equation system (DC2) for $\left(u, \psi, \varphi^{*}\right) \in \mathbb{X}^{D}$,

$$
\begin{array}{rlrl}
u+\mathcal{R} u-V_{\partial \Omega} \psi+W_{\Sigma} \varphi^{*} & =F_{0}^{D} & \text { in } \Omega_{\Sigma}, \\
\frac{1}{2} \psi+T^{+} \mathcal{R} u-\mathcal{W}_{\partial \Omega}^{\prime} \psi+T^{+} W_{\Sigma} \varphi^{*} & =T^{+} F_{0}^{D} & & \text { on } \partial \Omega,
\end{array}
$$




$$
T_{\Sigma}^{0} \mathcal{R} u-T_{\Sigma}^{0} V_{\partial \Omega} \psi+\mathcal{L}_{\Sigma}^{0} \varphi^{*}=T_{\Sigma}^{0} F_{0}^{D}-\psi_{\Sigma}^{0} \quad \text { on } \Sigma .
$$

Let us denote the left hand side operator of the BDIE system (DC2) as

$$
\mathcal{A}^{D 2}:=\left[\begin{array}{ccc}
I+\mathcal{R} & -V_{\partial \Omega} & W_{\Sigma} \\
T_{\partial \Omega}^{+} \mathcal{R} & \frac{1}{2} I-\mathcal{W}_{\partial \Omega}^{\prime} & T_{\partial \Omega}^{+} W_{\Sigma} \\
T_{\Sigma}^{0} \mathcal{R} & -T_{\Sigma}^{0} V_{\partial \Omega} & \mathcal{L}_{\Sigma}^{0}
\end{array}\right]
$$

where $T_{\partial \Omega}^{+}:=r_{\partial \Omega} T^{+}$.

Simplifying corresponding proofs of Theorems 4.1 and 4.2 , we arrive at the following equivalence theorem for the problem (DC) and BDIE systems (DC1) and (DC2), and the invertibility theorem for the operator $\mathcal{A}^{D 1}$ and $\mathcal{A}^{D 2}$.

THEOREM 5.1 Let conditions (2.17) hold.

(i) If a function $u \in H^{1}\left(\Omega_{\Sigma}\right)$ solves the problem $(D C)$, then the triple $\left(u, \psi, \varphi^{*}\right)$, where $\psi$ and $\varphi^{*}$ are defined by (5.1), solves BDIE systems (DC1) and (DC2).

(ii) If a triple $\left(u, \psi, \varphi^{*}\right) \in \mathbb{X}^{D}$ solves one of the BDIE systems (DC1) or (DC2), then this solution is unique and solves the both systems, while $u$ solves the problem $(D C)$ and relations (5.1) hold.

Let $\mathbb{F}^{+}:=H^{1}\left(\Omega_{\Sigma}\right) \times H^{\frac{1}{2}}(\partial \Omega) \times H^{-\frac{1}{2}}(\Sigma), \quad \mathbb{F}^{-}:=H^{1}\left(\Omega_{\Sigma}\right) \times H^{-\frac{1}{2}}(\partial \Omega) \times H^{-\frac{1}{2}}(\Sigma)$.

THEOREM 5.2 The operators $\mathcal{A}^{D 1}: \mathbb{X}^{D} \rightarrow \mathbb{F}^{+}$and $\mathcal{A}^{D 2}: \mathbb{X}^{D} \rightarrow \mathbb{F}^{-}$are continuous and continuously invertible.

\section{Segregated BDIEs for the problem (NC)}

Again, BDIE systems for problem (NC) are formulated by the same way as for the problem (MC) but with apparent simplifications. Let $u \in H^{1,0}\left(\Omega_{\Sigma} ; L\right)$ be a solution of the problem (NC) and let us introduce the notations

$$
\varphi=\gamma^{+} u \in H^{\frac{1}{2}}(\partial \Omega), \quad \varphi^{*}=[u]_{\Sigma} \in \widetilde{H}^{\frac{1}{2}}(\Sigma)
$$

for unknown boundary/crack values. Let $\mathbb{X}^{N}:=H^{1}\left(\Omega_{\Sigma}\right) \times H^{\frac{1}{2}}(\partial \Omega) \times \widetilde{H}^{\frac{1}{2}}(\Sigma)$.

BDIE system (NC1). Taking (3.11) in the domain, (3.13) on $\partial \Omega$ and (3.14) on $\Sigma$, substituting boundary/crack conditions (2.19)-(2.20) and employing (6.1), we derive the following boundary-domain integral equation system $(\mathrm{NC} 1)$ for $\left(u, \varphi, \varphi^{*}\right) \in \mathbb{X}^{N}$,

$$
\begin{array}{rlrl}
u+\mathcal{R} u+W_{\partial \Omega} \varphi+W_{\Sigma} \varphi^{*} & =F_{0}^{N} & \text { in } \Omega_{\Sigma}, \\
T^{+} \mathcal{R} u+\mathcal{L}_{\partial \Omega}^{+} \varphi+T^{+} W_{\Sigma} \varphi^{*} & =T^{+} F_{0}^{N}-\psi_{0} & & \text { on } \partial \Omega, \\
T_{\Sigma}^{0} \mathcal{R} u+T_{\Sigma}^{0} W_{\partial \Omega} \varphi+\mathcal{L}_{\Sigma}^{0} \varphi^{*} & =T_{\Sigma}^{0} F_{0}^{N}-\psi_{\Sigma}^{0} & & \text { on } \Sigma .
\end{array}
$$

where

$$
F_{0}^{N}:=\mathcal{P} f+V_{\Sigma}\left(\left[\psi_{\Sigma}\right]_{\Sigma}\right)+V_{\partial \Omega} \psi_{0} \quad \text { on } \quad \partial \Omega .
$$

Let us denote the left hand side operator of the BDIE system (NC1) as

$$
\mathcal{A}^{N 1}:=\left[\begin{array}{ccc}
I+\mathcal{R} & W_{\partial \Omega} & W_{\Sigma} \\
T_{\partial \Omega}^{+} \mathcal{R} & \mathcal{L}_{\partial \Omega}^{+} & T_{\partial \Omega}^{+} W_{\Sigma} \\
T_{\Sigma}^{0} \mathcal{R} & T_{\Sigma}^{0} W_{\partial \Omega} & \mathcal{L}_{\Sigma}^{0}
\end{array}\right] .
$$


BDIE system (NC2). Taking (3.11) in the domain, but (3.12) on $\partial \Omega$ and again (3.14) on $\Sigma$, substituting boundary/crack conditions (2.19)-(2.20) and employing (6.1), we derive the following boundary-domain integral equation system (NC2) for $\left(u, \varphi, \varphi^{*}\right) \in \mathbb{X}^{N}$,

$$
\begin{aligned}
u+\mathcal{R} u+W_{\partial \Omega} \varphi+W_{\Sigma} \varphi^{*} & =F_{0}^{N} & & \text { in } \Omega_{\Sigma}, \\
\frac{1}{2} \varphi+\gamma^{+} \mathcal{R} u+\mathcal{W}_{\partial \Omega} \varphi+\gamma^{+} W_{\Sigma} \varphi^{*} & =\gamma^{+} F_{0}^{N} & & \text { on } \partial \Omega, \\
T_{\Sigma}^{0} \mathcal{R} u+T_{\Sigma}^{0} W_{\partial \Omega} \varphi+\mathcal{L}_{\Sigma}^{0} \varphi^{*} & =T_{\Sigma}^{0} F_{0}^{N}-\psi_{\Sigma}^{0} & & \text { on } \Sigma .
\end{aligned}
$$

Let us denote the left hand side operator of the BDIE system (NC1) as

$$
\mathcal{A}^{N 2}:=\left[\begin{array}{ccc}
I+\mathcal{R} & W_{\partial \Omega} & W_{\Sigma} \\
\gamma_{\partial \Omega}^{+} \mathcal{R} & \frac{1}{2} I+\mathcal{W}_{\partial \Omega} & \gamma_{\partial \Omega}^{+} W_{\Sigma} \\
T_{\Sigma}^{0} \mathcal{R} & T_{\Sigma}^{0} W_{\partial \Omega} & \mathcal{L}_{\Sigma}^{0}
\end{array}\right]
$$

THEOREM 6.1 Let conditions (2.21) hold.

(i) If a function $u \in H^{1}\left(\Omega_{\Sigma}\right)$ solves the problem $(N C)$, then the triple $\left(u, \varphi, \varphi^{*}\right)$, where $\varphi$ and $\varphi^{*}$ are defined by (6.1), solves BDIE systems (NC1) and (NC2).

(ii) If a triple $\left(u, \varphi, \varphi^{*}\right) \in H^{1}\left(\Omega_{\Sigma}\right) \times H^{\frac{1}{2}}(\partial \Omega) \times \widetilde{H}^{\frac{1}{2}}(\Sigma)$ solves one of the BDIE system (NC1) or (NC2), then it solves the both systems, while u solves the problem (NC) and relations (6.1) hold.

(iii) Homogeneous BDIE systems (NC1) and (NC2) admit only one linearly independent solution $\left(u, \varphi, \varphi^{*}\right)=(1,1,0)$ in $\mathbb{X}^{N}$.

(iv) Condition (2.22) is necessary and sufficient for solvability of nonhomogeneous BDIE systems (NC1) and (NC2) in $\mathbb{X}^{N}$.

Proof. Items (i) and (ii) are obtained by simplifying the corresponding proof of Theorem 4.1. Then items (iii) and (iv) follow from items (i) and (ii) and from Theorem 2.1(iii), similar to the penultimate paragraph of the proof of Theorem 4.1.

THEOREM 6.2 The operators $\mathcal{A}^{N 1}: \mathbb{X}^{N} \rightarrow \mathbb{F}^{-}$and $\mathcal{A}^{N 2}: \mathbb{X}^{N} \rightarrow \mathbb{F}^{+}$are continuous Fredholm operators with zero index. They have one-dimensional null-space, $\operatorname{ker} \mathcal{A}^{N 1}=\operatorname{ker} \mathcal{A}^{N 2}$, spanned over the element $\left(u, \varphi, \varphi^{*}\right)=(1,1,0)$.

Proof. Let us consider the operators

$$
\mathcal{A}_{0}^{N 1}:=\left[\begin{array}{ccc}
I & W_{\partial \Omega} & W_{\Sigma} \\
0 & \hat{\mathcal{L}}_{\partial \Omega} & 0 \\
0 & 0 & \hat{\mathcal{L}}_{\Sigma}
\end{array}\right], \quad \mathcal{A}_{0}^{N 2}:=\left[\begin{array}{ccc}
I & W_{\partial \Omega} & W_{\Sigma} \\
0 & \frac{1}{2} I & 0 \\
0 & 0 & \hat{\mathcal{L}}_{\Sigma}
\end{array}\right] .
$$

It is evident from the Appendix that the operators $\mathcal{A}_{0}^{N 1}: \mathbb{X}^{N} \rightarrow \mathbb{F}^{-}$and $\mathcal{A}_{0}^{N 2}: \mathbb{X}^{N} \rightarrow \mathbb{F}^{+}$ are continuous, while the operators $\mathcal{A}^{N 1}-\mathcal{A}_{0}^{N 1}: \mathbb{X}^{N} \rightarrow \mathbb{F}^{-}$and $\mathcal{A}^{N 2}-\mathcal{A}_{0}^{N 2}: \mathbb{X}^{N} \rightarrow \mathbb{F}^{+}$are compact. By Theorem A.5 the operator $\hat{\mathcal{L}}_{\Sigma}: \widetilde{H}^{\frac{1}{2}}(\Sigma) \rightarrow H^{-\frac{1}{2}}(\Sigma)$ is continuously invertible and the operator $\hat{\mathcal{L}}_{\partial \Omega}: \widetilde{H}^{\frac{1}{2}}(\partial \Omega) \rightarrow H^{-\frac{1}{2}}(\partial \Omega)$ is a Fredholm operator with index zero. Then we conclude that the triangular operator $\mathcal{A}_{0}^{N 1}: \mathbb{X}^{N} \rightarrow \mathbb{F}^{-}$is a Fredholm operator with index zero, and the operator $\mathcal{A}_{0}^{N 2}: \mathbb{X}^{N} \rightarrow \mathbb{F}^{+}$is invertible. This implies that the operators $\mathcal{A}^{N 1}$ : $\mathbb{X}^{N} \rightarrow \mathbb{F}^{-}$and $\mathcal{A}^{N 2}: \mathbb{X}^{N} \rightarrow \mathbb{F}^{+}$are Fredholm operators with index zero. By Theorem 6.1(iii), $\operatorname{ker} \mathcal{A}^{N 1}=\operatorname{ker} \mathcal{A}^{N 2}$ is one-dimensional and is spanned over the element $\left(u, \varphi, \varphi^{*}\right)=(1,1,0)$. 


\section{Concluding remarks}

For a scalar "Laplace" PDE with variable coefficient on a three-dimensional bounded domain with a crack, segregated direct boundary-domain integral equation systems associated with the Dirichlet, Neumann and mixed boundary conditions on the external boundary and Neumann conditions on the crack faces, have been formulated and analysed in the paper. Among these, four different BDIE systems were given for the mixed problem, two for the Dirichlet and two for the Neumann problems. Equivalence of the BDIE systems to the original BVPs was proved in the case when right-hand side of the PDE is from $L_{2}(\Omega)$, and the Dirichlet and the Neumann data from the spaces $H^{\frac{1}{2}}\left(S_{D}\right)$ and $H^{-\frac{1}{2}}\left(S_{N}\right), \mathbb{H}^{-\frac{1}{2}}(\Sigma)$, respectively. The invertibility of the BDIE operators was proved in the corresponding Sobolev spaces.

Using approach of [13] united direct boundary-domain integro-differential systems can be also formulated and analysed for the BVPs with crack. The BDIEs for unbounded domains with cracks can be analysed as well. The approach can be extended also to more general PDEs and to systems of PDEs, while smoothness of the variable coefficients and the boundary can be essentially relaxed, and the PDE right hand side can be considered in more general spaces, c.f. $[12,13]$.

Employing methods of [1] and [4], one can consider also the localised counterparts of the BDIEs for BVPs with cracks.

\section{Acknowledgements}

This work was supported by the International Joint Project Grant - 2005/R4 "BoundaryDomain Integral Equations: Formulation, Analysis, Localisation" of the Royal Society, UK, and the grant "Mathematical analysis of localised boundary-domain integral equations for BVPs with variable coefficients" of the EPSRC, UK.

\section{APPENDIX}

\section{A Properties of surface potentials}

The auxiliary facts collected in Theorems A.1-A.5 follow e.g. from [5, Theorem 2], [6, Ch. XI, Part B, §3,], [17, Theorem 2.7(ii)], [2, Section 3], [13, Appendix].

THEOREM A.1 The following operators are continuous,

$$
\begin{array}{cl}
V_{\partial \Omega}: H^{-\frac{1}{2}}(\partial \Omega) \rightarrow H^{1,0}(\Omega ; L), & W_{\partial \Omega}: H^{\frac{1}{2}}(\partial \Omega) \rightarrow H^{1,0}(\Omega ; L), \\
V_{\Sigma}: \widetilde{H}^{-\frac{1}{2}}(\Sigma) \rightarrow H^{1,0}\left(\Omega_{\Sigma} ; L\right), & W_{\Sigma}: \widetilde{H}^{\frac{1}{2}}(\Sigma) \rightarrow H^{1,0}\left(\Omega_{\Sigma} ; L\right) .
\end{array}
$$

THEOREM A.2 The following operators are continuous.

$$
\begin{array}{cl}
\mathcal{V}_{\partial \Omega}: H^{-\frac{1}{2}}(\partial \Omega) \rightarrow H^{\frac{1}{2}}(\partial \Omega), & \mathcal{V}_{\Sigma}: \widetilde{H}^{-\frac{1}{2}}(\Sigma) \rightarrow H^{\frac{1}{2}}(\Sigma), \\
\mathcal{W}_{\partial \Omega}: H^{\frac{1}{2}}(\partial \Omega) \rightarrow H^{\frac{1}{2}}(\partial \Omega), & \mathcal{W}_{\Sigma}: \widetilde{H}^{\frac{1}{2}}(\Sigma) \rightarrow H^{\frac{1}{2}}(\Sigma), \\
\mathcal{W}_{\partial \Omega}^{\prime}: H^{-\frac{1}{2}}(\partial \Omega) \rightarrow H^{-\frac{1}{2}}(\partial \Omega), & \mathcal{W}_{\Sigma}^{\prime}: \widetilde{H}^{-\frac{1}{2}}(\Sigma) \rightarrow H^{-\frac{1}{2}}(\Sigma), \\
\mathcal{L}_{\partial \Omega}^{ \pm}: H^{\frac{1}{2}}(\partial \Omega) \rightarrow H^{-\frac{1}{2}}(\partial \Omega), & \mathcal{L}_{\Sigma}^{ \pm}: \widetilde{H}^{\frac{1}{2}}(\Sigma) \rightarrow H^{-\frac{1}{2}}(\Sigma)
\end{array}
$$

Moreover the operators (A.2)-(A.3) are compact. 
THEOREM A.3 Let $\varphi \in H^{\frac{1}{2}}(\partial \Omega), \psi \in H^{-\frac{1}{2}}(\partial \Omega), \psi^{*} \in \widetilde{H}^{-\frac{1}{2}}(\Sigma)$ and $\varphi^{*} \in \widetilde{H}^{\frac{1}{2}}(\Sigma)$. Then there hold the following jump relations on $\partial \Omega$,

$$
\begin{aligned}
& \gamma^{ \pm} V_{\partial \Omega} \psi=\mathcal{V}_{\partial \Omega} \psi \\
& \gamma^{ \pm} W_{\partial \Omega} \varphi=\mp \frac{1}{2} \varphi+\mathcal{W}_{\partial \Omega} \varphi \\
& T^{ \pm} V_{\partial \Omega} \psi= \pm \frac{1}{2} \psi+\mathcal{W}_{\partial \Omega}^{\prime} \psi \\
& T^{+} W_{\partial \Omega} \varphi-T^{-} W_{\partial \Omega} \varphi=\mathcal{L}_{\partial \Omega}^{+} \varphi-\mathcal{L}_{\partial \Omega}^{-} \varphi=\frac{\partial a}{\partial n} \varphi
\end{aligned}
$$

and similar jump relations on $\Sigma$,

$$
\begin{aligned}
& \gamma^{ \pm} V_{\Sigma} \psi^{*}=\mathcal{V}_{\Sigma} \psi^{*}, \\
& \gamma^{ \pm} W_{\Sigma} \varphi^{*}=\mp \frac{1}{2} \varphi^{*}+\mathcal{W}_{\Sigma} \varphi^{*}, \\
& T^{ \pm} V_{\Sigma} \psi^{*}= \pm \frac{1}{2} \psi^{*}+\mathcal{W}_{\Sigma}^{\prime} \psi^{*}, \\
& T^{+} W_{\Sigma} \varphi^{*}-T^{-} W_{\Sigma} \varphi^{*}=\mathcal{L}_{\Sigma}^{+} \varphi^{*}-\mathcal{L}_{\Sigma}^{-} \varphi^{*}=\frac{\partial a}{\partial n} \varphi^{*} .
\end{aligned}
$$

THEOREM A.4 Let $S$ be a nonempty, simply connected sub-manifold of $\partial \Omega$ with infinitely smooth boundary curve. Then the operators

$$
\mathcal{V}_{\partial \Omega}: H^{-\frac{1}{2}}(\partial \Omega) \rightarrow H^{\frac{1}{2}}(\partial \Omega), \quad r_{S} \mathcal{V}_{\partial \Omega}: \widetilde{H}^{-\frac{1}{2}}(S) \rightarrow H^{\frac{1}{2}}(S)
$$

are continuously invertible.

THEOREM A.5 Let the operator $\hat{\mathcal{L}}_{S}$ be given by (4.26).

(i) The operator $\hat{\mathcal{L}}_{\partial \Omega}: H^{\frac{1}{2}}(\partial \Omega) \rightarrow H^{-\frac{1}{2}}(\partial \Omega)$ is Fredholm operator with zero index and the operator $\mathcal{L}_{\partial \Omega}^{ \pm}-\hat{\mathcal{L}}_{\partial \Omega}: H^{\frac{1}{2}}(\partial \Omega) \rightarrow H^{-\frac{1}{2}}(\partial \Omega)$ is compact.

(ii) Let $S=\Sigma$ or $S$ along with $\partial \Omega \backslash \bar{S}$ be nonempty, open simply connected sub-manifolds of $\partial \Omega$ with an infinitely smooth boundary curve. Then the operator $\hat{\mathcal{L}}_{S}: \widetilde{H}^{\frac{1}{2}}(S) \rightarrow H^{-\frac{1}{2}}(S)$ is continuously invertible and the operator $\mathcal{L}_{S}^{ \pm}-\hat{\mathcal{L}}_{S}: \widetilde{H}^{\frac{1}{2}}(S) \rightarrow H^{-\frac{1}{2}}(S)$ is compact.

THEOREM A.6 The following operators are compact,

$$
\begin{array}{cc}
\gamma_{\Sigma}^{ \pm} V_{\partial \Omega}: H^{-\frac{1}{2}}(\partial \Omega) \rightarrow H^{\frac{1}{2}}(\Sigma), & \gamma_{\Sigma}^{ \pm} W_{\partial \Omega}: H^{\frac{1}{2}}(\partial \Omega) \rightarrow H^{\frac{1}{2}}(\Sigma), \\
T_{\Sigma}^{ \pm} V_{\partial \Omega}: H^{-\frac{1}{2}}(\partial \Omega) \rightarrow H^{-\frac{1}{2}}(\Sigma), & T_{\Sigma}^{ \pm} W_{\partial \Omega}: H^{\frac{1}{2}}(\partial \Omega) \rightarrow H^{-\frac{1}{2}}(\Sigma), \\
\gamma_{\partial \Omega}^{+} V_{\Sigma}: \widetilde{H}^{-\frac{1}{2}}(\Sigma) \rightarrow H^{\frac{1}{2}}(\partial \Omega), & \gamma_{\partial \Omega}^{+} W_{\Sigma}: \widetilde{H}^{\frac{1}{2}}(\Sigma) \rightarrow H^{\frac{1}{2}}(\partial \Omega), \\
T_{\partial \Omega}^{+} V_{\Sigma}: \widetilde{H}^{-\frac{1}{2}}(\Sigma) \rightarrow H^{\frac{1}{2}}(\partial \Omega), & T_{\partial \Omega}^{+} W_{\Sigma}: \widetilde{H}^{\frac{1}{2}}(\Sigma) \rightarrow H^{\frac{1}{2}}(\partial \Omega) .
\end{array}
$$

Proof. Let $S$ be either $\partial \Omega$ or $\Sigma$. As shown in [2],

$$
V_{S} g=\frac{1}{a} V_{\Delta, S} g, \quad W_{S} g=\frac{1}{a} W_{\Delta, S}(a g),
$$

where $V_{\Delta, S}, W_{\Delta, S}$ are the single and double layer potentials for the Laplace operator, with the densities having support on $S$. Since $\Delta V_{\Delta, S} g=0, \Delta W_{\Delta, S} g=0$ on $\mathbb{R}^{3} \backslash S$, we have $V_{\Delta, S} g, W_{\Delta, S} g \in C^{\infty}\left(\mathbb{R}^{3} \backslash S\right)$ by the solution regularity theorem for strongly elliptic equations, see e.g. [9], which by (A.13) implies also $V_{S} g, W_{S} g \in C^{\infty}\left(\mathbb{R}^{3} \backslash S\right)$. Since $\partial \Omega$ and $\Sigma$ do not intersect, employing the Rellich compact imbedding theorem completes the proof. 


\section{B Properties of volume potentials}

THEOREM B.1 The following operators are continuous

$$
\begin{aligned}
& \mathcal{P}: H^{0}(\Omega) \rightarrow H^{2}(\Omega) \subset H^{1,0}(\Omega ; L), \\
& \mathcal{R} \quad: \quad H^{0}(\Omega) \rightarrow H^{1}(\Omega), \\
&: \quad H^{1}\left(\Omega_{\Sigma}\right) \rightarrow H^{1,0}\left(\Omega_{\Sigma} ; L\right) .
\end{aligned}
$$

Moreover, for $f \in H^{0}\left(\Omega_{\Sigma}\right)$ and $u \in H^{1}\left(\Omega_{\Sigma}\right)$ we have,

$$
[\mathcal{P} f]_{\Sigma}=0, \quad[T]_{\Sigma} \mathcal{P} f=0, \quad[\mathcal{R} u]_{\Sigma}=0, \quad[T]_{\Sigma} \mathcal{R} u=-\left(\partial_{n} a\right)[u]_{\Sigma} .
$$

Proof. The continuity of operators (B.1) and (B.2) is proved in [2, Theorem 3.8]. Similar to the proof of [2, Theorem 3.8], integrating by parts we have the following relation for $g \in H^{1}\left(\Omega_{\Sigma}\right)$

$$
\partial_{j} \mathcal{P}_{\Delta} g=\mathcal{P}_{\Delta}\left(\partial_{j} g\right)+V_{\Delta, \partial \Omega}\left(n_{j} \gamma^{+} g\right)+V_{\Delta, \Sigma}\left(n_{j}[g]_{\Sigma}\right) \quad \text { in } \Omega_{\Sigma} .
$$

Taking into account that expressions (3.1) and (3.3) give

$$
\mathcal{R} u=-\frac{1}{a} \sum_{j=1}^{3} \partial_{j}\left[\mathcal{P}_{\Delta}\left(u \partial_{j} a\right)\right]
$$

we have from (B.5),

$$
\mathcal{R} u=-\frac{1}{a} \sum_{j=1}^{3} \mathcal{P}_{\Delta} \partial_{j}\left(u \partial_{j} a\right)-V_{\Delta, \partial \Omega}\left(\gamma^{+} u \partial_{n} a\right)-V_{\Delta, \Sigma}\left([u]_{\Sigma} \partial_{n} a\right) \quad \text { in } \Omega_{\Sigma}
$$

which along with (B.1) and Theorem A.1 implies (B.3). The first two relations in (B.4) follow from (B.1) and imply the last two by (B.6) and Theorem A.3.

The following statement is implied by [2, Corollary 3.9].

THEOREM B.2 Let $S=\partial \Omega$, or $S$ be a nonempty, open sub-manifold $\partial \Omega$ with an infinitely smooth boundary curve, or $S=\Sigma$. The operators

$$
\begin{aligned}
\mathcal{R} & : \quad H^{1}\left(\Omega_{\Sigma}\right) \rightarrow H^{1}\left(\Omega_{\Sigma}\right), \\
r_{S} \gamma^{ \pm} \mathcal{R} & : \quad H^{1}\left(\Omega_{\Sigma}\right) \rightarrow H^{\frac{1}{2}}(S), \\
r_{S} T^{ \pm} \mathcal{R} & : \quad H^{1}\left(\Omega_{\Sigma}\right) \rightarrow H^{-\frac{1}{2}}(S)
\end{aligned}
$$

are compact.

\section{References}

[1] O. Chkadua, S. E. Mikhailov, and D. Natroshvili. About analysis of some localized boundary-domain integral equations for a variable-coefficient BVPs. In J. Trevelyan, editor, Advances in Boundary Integral Methods - Proceedings of the Sixth UK Conference on Boundary Integral Methods, pages 291-302. Durham University Publ., UK, 2007.

[2] O. Chkadua, S. E. Mikhailov, and D. Natroshvili. Analysis of direct boundary-domain integral equations for a mixed BVP with variable coefficient, I: Equivalence and invertibility. Journal of Integral Equations and Applications, 21(4):499-543, 2009. 
[3] O. Chkadua, S. E. Mikhailov, and D. Natroshvili. Analysis of some boundary-domain integral equations for variable-coefficient problems with cracks. In H. Power, A. L. Rocca, and S. Baxter, editors, Proceedings of the 7th UK Conference on Boundary Integral Methods, UKBIM7, pages 37-51. University of Nottingham, UK, 2009.

[4] O. Chkadua, S. E. Mikhailov, and D. Natroshvili. Analysis of some localized boundarydomain integral equations. Journal of Integral Equations and Applications, 21:405-445, 2009.

[5] M. Costabel. Boundary integral operators on Lipschitz domains: elementary results. SIAM J. Math. Anal., 19:613-626, 1988.

[6] R. Dautray and J. Lions. Mathematical Analysis and Numerical Methods for Science and Technology, volume 4: Integral Equations and Numerical Methods. Springer, BerlinHeidelberg-New York, 1990.

[7] P. Grisvard. Elliptic Problems in Nonsmooth Domains. Pitman, Boston-LondonMelbourne, 1985.

[8] G. Hsiao and W. Wendland. Boundary Integral Equations. Springer, Berlin - Heidelberg, 2008.

[9] J.-L. Lions and E. Magenes. Non-Homogeneous Boundary Value Problems and Applications, volume 1. Springer, Berlin - Heidelberg - New York, 1972.

[10] W. McLean. Strongly Elliptic Systems and Boundary Integral Equations. Cambridge University Press, Cambridge, UK, 2000.

[11] S. E. Mikhailov. Localized boundary-domain integral formulations for problems with variable coefficients. Engineering Analysis with Boundary Elements, 26:681-690, 2002.

[12] S. E. Mikhailov. Analysis of extended boundary-domain integral and integro-differential equations of some variable-coefficient BVP. In K. Chen, editor, Advances in Boundary Integral Methods - Proceedings of the 5th UK Conference on Boundary Integral Methods, pages 106-125, Liverpool, UK, 2005. University of Liverpool Publ.

[13] S. E. Mikhailov. Analysis of united boundary-domain integro-differential and integral equations for a mixed BVP with variable coefficient. Math. Methods in Applied Sciences, 29:715-739, 2006.

[14] S. E. Mikhailov. About traces, extensions and co-normal derivative operators on Lipschitz domains. In C. Constanda and S. Potapenko, editors, Integral Methods in Science and Engineering: Techniques and Applications, pages 149-160. Birkhäuser, Boston, 2008.

[15] C. Miranda. Partial Differential Equations of Elliptic Type. Springer, Berlin - Heidelberg - New York, 2-nd edition, 1970.

[16] A. Pomp. The boundary-domain integral methid for elliptic systems. With applications in shells, volume 1683 of Lecture Notes in Mathematics. Springer, Berlin - Heidelberg New York, 1998.

[17] E. Stephan. Boundary integral equations for screen problems in $\mathbb{R}^{3}$. Integral Equations and Operator Theory, 10:236-257, 1987. 